\title{
Drug Accountability Completion Status
}

National Cancer Institute

\section{Source}

National Cancer Institute. Drug Accountability Completion Status. NCI Thesaurus. Code C113357.

A term used to describe the state or condition of the completeness of the drug accountability data. 